Bio - grafia. Escritos sobre la Biología y su Enseñanza. ISSN 2027

Edición Extraordinaria. p.p. 1543-1563

Memorias del VIII Encuentro Nacional de Experiencias en Enseñanza de la Biología y la Educación Ambiental. III Congreso Nacional de Investigación en Enseñanza de la Biología.

\title{
PREVENCIÓN DEL CONSUMO DE CANNABIS SATIVA A PARTIR DE LA ENSEÑANZA DEL SISTEMA NERVIOSO. Cenaida Fajardo Rodríguez ${ }^{1}$
}

En el colegio Domingo Faustino Sarmiento IED, el consumo de Cannabis sativa es un problema que día a día crece igual que va aumentando su uso en la población juvenil colombiana.

Buscando alternativas de solución ante el crecimiento de consumidores precoces de cannabis, se creó una propuesta didáctica para la prevención del consumo de cannabis sativa a partir de la enseñanza del sistema nervioso, con los estudiantes del grado noveno, en donde se tiene como meta de comprensión: los estudiantes comprenderán los efectos del consumo de Cannabis sativa en el organismo mediante el estudio del sistema nervioso con el fin de generar posturas críticas y decisiones responsables a nivel individual y colectivo.

El proceso de investigación implementó la enseñanza para la comprensión generando desempeños que promueven en el estudiante el desarrollo de actitudes científicas que permiten reconocer en su entorno necesidades de la vida cotidiana para que desde sus intereses y saberes puedan contribuir con la solución de este flagelo institucional y social.

N

c

I

A

E

Con la recopilación de proyectos de sintesis en cada uno de los encuentros de clase, los grupos de trabajo generaron alternativas para sensibilizar y prevenir el consumo de sustancias psicoactivas en los diferentes estamentos de la comunidad educativa como son: un plegable, un video, una obra de títeres y talleres reflexivos con cartas anónimas, elaboradas por ellos mismos donde argumentan por qué rechazar el consumo de cannabis.

PALABRAS CLAVE: prevención del consumo de cannabis, sistema nervioso, enseñanza para la comprensión.

ABSTRACT

${ }^{1}$ Docente del Colegio Técnico Domingo Faustino Sarmiento. IED.
Cenafajardo_7@hotmail.com

1543 
Bio - grafia. Escritos sobre la Biología y su Enseñanza. ISSN 2027

Edición Extraordinaria. p.p. 1543-1563

Memorias del VIII Encuentro Nacional de Experiencias en Enseñanza de la Biología y la Educación Ambiental. III Congreso Nacional de Investigación en Enseñanza de la Biología.

\section{USE PREVENTION OF CANNABIS SATIVA TEACHING FROM NERVOUS SYSTEM.}

At school Domingo Faustino Sarmiento FDI, cannabis sativa is a problem that grows daily as increases its use in Colombian youth population.

You looking for alternative solutions to the growth of early cannabis, a didactic approach for the prevention of cannabis sativa from the teaching of the nervous system, with students from ninth grade is created, where it aims to understand: Students will understand the effects of cannabis sativa in the body through the study of the nervous system in order to generate critical positions and responsible decisions at individual and collective level.

The research process implements generating teaching for understanding performance in promoting the development of scientific student attitudes to recognize in their environment needs of everyday life since their interests and knowledge can contribute to solving this scourge and institutional social.

With the compilation of draft synthesis in each class meetings, working groups generated alternatives to raise awareness and prevent the use of psychoactive substances in different sectors of the education community, such as: a folding, a video, a work Puppets and reflective workshops with anonymous letters, which made themselves argue why reject cannabis.

KEYWORDS: Prevention of cannabis, nervous system, teaching for understanding.

\section{INTRODUCCIÓN}

El Colegio Domingo Faustino Sarmiento. I.E.D se encuentra ubicado en el barrio Rionegro localidad (12) Barrios Unidos. Presta el servicio educativo a cerca de 1800 estudiantes desde el ciclo de primera infancia hasta el bachillerato. Es de carácter técnico con especialidades en: Operaciones comerciales y financieras y organización de eventos recreodeportivos. 
Bio - grafia. Escritos sobre la Biología y su Enseñanza. ISSN 2027

Edición Extraordinaria. p.p. 1543-1563

Memorias del VIII Encuentro Nacional de Experiencias en Enseñanza de la Biología y la Educación Ambiental. III Congreso Nacional de Investigación en Enseñanza de la Biología.

La misión institucional expresa: Colegio técnico que trasciende con educación de calidad; para construir y vivenciar con los estudiantes procesos pedagógicos para su desarrollo humano, mediante la constante motivación por el conocimiento y el cultivo de principios y valores, que les permite forjar su proyecto de vida e interactuar con su entorno, en niveles más cualificados de autonomía, creatividad y espíritu emprendedor.

Existen razones para trabajar esta temática, una de ellas es que en el colegio hasta el año 2004 no se registraba estudiantes consumidores de cannabis, pero años después, han venido aumentando el número de educandos consumidores.

En la ley 1098 de 2006: La Ley de Infancia y Adolescencia, asigna la obligación ética a los establecimientos educativos de prevenir el tráfico y consumo de todo tipo de sustancias psicoactivas que producen dependencia dentro de las instalaciones educativas y solicitar a las autoridades competentes acciones efectivas contra el tráfico, venta y consumo alrededor de las instalaciones educativas (artículo 43, numeral 7). Otra razón que motiva la investigación.

El consumo de drogas de abuso en Colombia, ha aumentado de manera significativa en los últimos años, entre 1998 y 2006 (Scopetta, 2010). La UNODC reporta que la edad de inicio de consumo de marihuana por los adolescentes colombianos se da hacia los 14 años. Un factor importante a tener en cuenta es que la población estudiantil, principalmente universitaria y de educación secundaria es la que se ve más afectada por esta problemática, de acuerdo a diferentes informes sobre tendencias de consumo de drogas en Colombia y América Latina y estudios como el de Beleño y Díaz (2011). Por lo que la escuela se convierte en un lugar privilegiado para la intervención sobre esta problemática.

En el marco legal educativo del país, desde los planteamientos de la didáctica de la biología se plantea que el objetivo de un docente no se reduce a enseñar biología, sino que también incluye formar ciudadanas y ciudadanos críticos, capaces de resolver problemas y participar en la toma de decisiones que involucren a toda una comunidad en general (Jiménez, 2003). Buscando que el conocimiento construido dentro del aula de clase, pueda 
Bio - grafia. Escritos sobre la Biología y su Enseñanza. ISSN 2027

Edición Extraordinaria. p.p. 1543-1563

Memorias del VIII Encuentro Nacional de Experiencias en Enseñanza de la Biología y la Educación Ambiental. III Congreso Nacional de Investigación en Enseñanza de la Biología.

trascender y aplicarse a la solución de los problemas de la vida cotidiana de los estudiantes. Bajo esta perspectiva, el propósito del proceso educativo se transforma, por lo que "el aprendizaje le representará al estudiante un beneficio que le proporcione un arsenal de herramientas para comprender su mundo y su experiencia en él, así como para orientar su acción de una manera creativa y responsable" (Escobedo; et al, 2004).

En el proyecto de investigación: Prevención del consumo de sustancias psicoactivas a través de la implementación de una propuesta didáctica de la enseñanza de la biología que aporte a la solución de problemas sociales a partir de los resultados de la investigación: "Efectos del consumo del THC (delta-9-tetrahidrocanabinol) en el sistema nervioso, circulatorio, respiratorio, e influencia en la capacidad cognitiva, de consumidores universitarios habituales" (Moreno; et al, 2013), propuesto por los grupos de investigación Neurociencia GINUD y de investigación Biología Enseñanza y realidades BER de la Universidad Distrital; se acepta la invitación y se decide trabajar con el grado noveno, con el enfoque pedagógico Enseñanza para la Comprensión (E.p.C) bajo el Hilo Conductor o meta de comprensión abarcadora (es lo que se busca comprender en la unidad, el sueño que se quiere lograr): Efectos del consumo de Cannabis sativa en el organismo mediante el estudio del sistema nervioso con el fin de generar posturas críticas y decisiones responsables a nivel individual y colectivo.

El objetivo general es implementar una propuesta didáctica que aporte a la apropiación y toma de decisiones frente a la problemática de consumo de Cannabis sativa a partir de la comprensión del concepto de sistema nervioso. Los objetivos específicos son: realizar una caracterización de ideas previas de los estudiantes acerca del sistema nervioso y de la cannabis, diseñar una propuesta didáctica para la enseñanza del sistema nervioso a partir de los efectos del consumo de $C$. sativa y participar en la construcción de estrategias que incluyan resultados de la aplicación de la propuesta didáctica para prevenir el consumo de Cannabis.

METODOLOGÍA 
Bio - grafia. Escritos sobre la Biología y su Enseñanza. ISSN 2027

Edición Extraordinaria. p.p. 1543-1563

Memorias del VIII Encuentro Nacional de Experiencias en Enseñanza de la Biología y la Educación Ambiental. III Congreso Nacional de Investigación en Enseñanza de la Biología.

Para entender el concepto de enseñanza para la comprensión (E.p.C), es necesario remitirse al concepto de comprensión, en palabras de Perkins, "La comprensión se presenta cuando la gente puede pensar y actuar con flexibilidad a partir de lo que sabe. Por contraste, cuando un estudiante no puede ir más allá de la memorización y el pensamiento y la acción rutinarios, esto indica falta de comprensión" (1999). De esta manera se espera que en el aula no se busque más que una simple repetición de los conceptos trabajados, sino la apropiación por parte de los estudiantes, de manera que estos conocimientos obtenidos puedan ser explicados por ellos mismos y sean aplicables a diversas situaciones.

Aprovechando las reflexiones sobre qué debemos enseñar, qué vale la pena comprender, cómo se debe enseñar para lograr comprensiones y cómo verificar esas comprensiones tanto por parte de los estudiantes como del docente se construye la ruta de trabajo, basada en los elementos de la enseñanza para la comprensión, como son: tópico generativo, metas de comprensión, desempeños de comprensión y evaluación continua.

El proceso metodológico presentó tres etapas:

Primera etapa: Organización de grupos de trabajo y elaboración de acuerdos. Diagnóstico de factores de riesgo asociados al consumo de $C$. sativa a través de la elaboración de un mapa del colegio y zonas aledañas, en las cuales identificaron con íconos algunos problemas y a la vez daban respuesta a preguntas como: qué problemas (dos) presenta el colegio, cómo se originan, cómo afectan la calidad de vida, a futuro cómo ve el problema, a quien corresponde solucionar el problema, qué papel tiene la escuela en la solución del problema y cómo aporta la clase de biología en la solución del problema? Se aplicó también, una encuesta a 165 estudiantes de los grados $6^{\circ}, 9^{\circ}, 10^{\circ}$ y $11^{\circ}$ sobre el conocimiento de sustancias psicoactivas (SPA). (Anexo 1). Además se realizó un taller sobre la importancia del estudio del sistema nervioso en la prevención del consumo de $C$ sativa. (Anexo 2).

Segunda etapa: Se trabajaron los tópicos generativos relacionados con el sistema nervioso. Importancia del estudio del sistema nervioso en la prevención del consumo de $C$. sativa. Anatomía y fisiología el sistema nervioso. Relaciones del sistema nervioso con los otros sistemas del cuerpo humano. Sistema límbico y neurotransmisores. La planta $C$. Sativa y los 
Bio - grafia. Escritos sobre la Biología y su Enseñanza. ISSN 2027

Edición Extraordinaria. p.p. 1543-1563

Memorias del VIII Encuentro Nacional de Experiencias en Enseñanza de la Biología y la Educación Ambiental. III Congreso Nacional de Investigación en Enseñanza de la Biología.

Cannabinoides. Caracteres taxonómicos de la Cannabis. Clasificación científica de la marihuana. Efectos por el consumo y prevención del consumo de cannabis.

Tercera Etapa: construcción de propuestas sobre la pregunta ¿Cómo sensibilizar a la comunidad DOFASA para evitar el consumo de Cannabis sativa?

\section{RESULTADOS Y DISCUSIÓN.}

Desde el comienzo de la investigación los estudiantes se organizaron libremente, acordaron un nombre que identificaran al grupo y se acordó que como grupo todos iban a compartir responsabilidades en cuanto a consecución de materiales, digitación de la bitácora que contenía datos como: fecha, lugar, sesión, nombre del grupo, tópico generativo, desempeño de comprensión, narración de la actividad, proyecto síntesis y compromisos.

En la primera actividad de diagnóstico los estudiantes reconocieron problemas el hostigamiento escolar, la producción de basuras, los robos, el mal uso de los baños y bienes del colegio y por su puesto el consumo de cannabis. Cada una de estas temáticas se les dio importancia al integrarlas en los núcleos temáticos ecosistémico y organísmico propios del área, como también, incluirlas en la dimensión físico-creativa, socio-afectiva porque contribuyen a fortalecer los ejes institucionales de comunicación, emprendimiento, convivencia y ciudadanía, aportando elementos y criterios en la construcción del proyecto de vida de los estudiantes

La siguiente tabla indica información derivada del diagnóstico sobre la marihuana, origen del consumo, cómo afecta la calidad de vida escolar y cuál es el papel de la escuela frente al problema.

Tabla 1. Percepciones de los estudiantes sobre la marihuana.

\begin{tabular}{|l|l|l|}
\hline ORIGEN DEL CONSUMO & $\begin{array}{l}\text { COMO AFECTA LA } \\
\text { CALIDAD DE VIDA }\end{array}$ & PAPEL DE LA ESCUELA \\
\hline Por curiosidad se aceptan & $\begin{array}{l}\text { Se tiene que aguantar el } \\
\text { olor a marihuana en los }\end{array}$ & $\begin{array}{l}\text { Capacitar sobre el } \\
\text { conocimiento del daño que }\end{array}$ \\
\hline
\end{tabular}

1548 
Bio - grafia. Escritos sobre la Biología y su Enseñanza. ISSN 2027

Edición Extraordinaria. p.p. 1543-1563

Memorias del VIII Encuentro Nacional de Experiencias en Enseñanza de la Biología y la Educación Ambiental. III Congreso Nacional de Investigación en Enseñanza de la Biología.

\begin{tabular}{|l|l|l|}
\hline la probadita & baños. & producen estas SPA. \\
\hline $\begin{array}{l}\text { Por malas influencias fuera } \\
\text { y dentro del colegio }\end{array}$ & $\begin{array}{l}\text { Cambia de aspecto físico } \\
\text { (flaco, loco, desesperado) } \\
\text { y lenguaje. Se vuelven } \\
\text { agresivos. Cambia de } \\
\text { amigos. }\end{array}$ & $\begin{array}{l}\text { Realizar proyectos que } \\
\text { motiven la prevención del } \\
\text { consumo. Requisas } \\
\text { permanentes. }\end{array}$ \\
\hline $\begin{array}{l}\text { Falta de autoestima, } \\
\text { permite dejarse } \\
\text { influenciar. No saber decir } \\
\text { "NO" }\end{array}$ & $\begin{array}{l}\text { Se puede perder } \\
\text { amistades, el cupo, el año } \\
\text { de estudio. } \\
\text { Pueden cambiar su } \\
\text { vestuario y aderezos. }\end{array}$ & $\begin{array}{l}\text { Reflexiones por parte de } \\
\text { orientación sobre la } \\
\text { relación del consumo de } \\
\text { drogas y cambios } \\
\text { psicológicos y sociales en } \\
\text { el estudiante y familia }\end{array}$ \\
\hline $\begin{array}{l}\text { Como escape a problemas } \\
\text { familiares. } \\
\text { Por llamar la atención. }\end{array}$ & $\begin{array}{l}\text { Sentimientos de soledad, } \\
\text { fracaso, aislamiento. } \\
\text { Perder a la familia, amigos } \\
\text { e inclusive el trabajo }\end{array}$ & $\begin{array}{l}\text { Capacitar a los padres de } \\
\text { familia en el tema. }\end{array}$ \\
\hline Por ignorancia & $\begin{array}{l}\text { Caer en el problema. } \\
\text { Enloquecer a los jóvenes. }\end{array}$ & $\begin{array}{l}\text { Trabajar sobre proyecto } \\
\text { de vida }\end{array}$ \\
\hline $\begin{array}{l}\text { No se tienen claros los } \\
\text { sueños }\end{array}$ & $\begin{array}{l}\text { Sentimientos de fracaso, } \\
\text { deserción y suicidio. }\end{array}$ & $\begin{array}{l}\text { Trabajar sobre la } \\
\text { corresponsabilidad } \\
\text { problema. }\end{array}$ \\
$\begin{array}{l}\text { Familia-escuela } \\
\text { aumenta el número de } \\
\text { consumidores y puede } \\
\text { involucrarse la seguridad } \\
\text { de muchos otros }\end{array}$ & $\begin{array}{l}\text { Protagonista para } \\
\text { procesos de prevención y } \\
\text { control. }\end{array}$ \\
\hline
\end{tabular}


Bio - grafia. Escritos sobre la Biología y su Enseñanza. ISSN 2027

Edición Extraordinaria. p.p. 1543-1563

Memorias del VIII Encuentro Nacional de Experiencias en Enseñanza de la Biología y la Educación Ambiental. III Congreso Nacional de Investigación en Enseñanza de la Biología.

estudiantes

Esta información se relaciona con los datos logrados en la encuesta aplicada a estudiantes de cuatro grados. A la pregunta con cuáles de las siguientes situaciones asocia el consumo de sustancias psicoactivas en niños y jóvenes? Respondieron así:

Gráfico uno. Situaciones asociadas al consumo de SPA

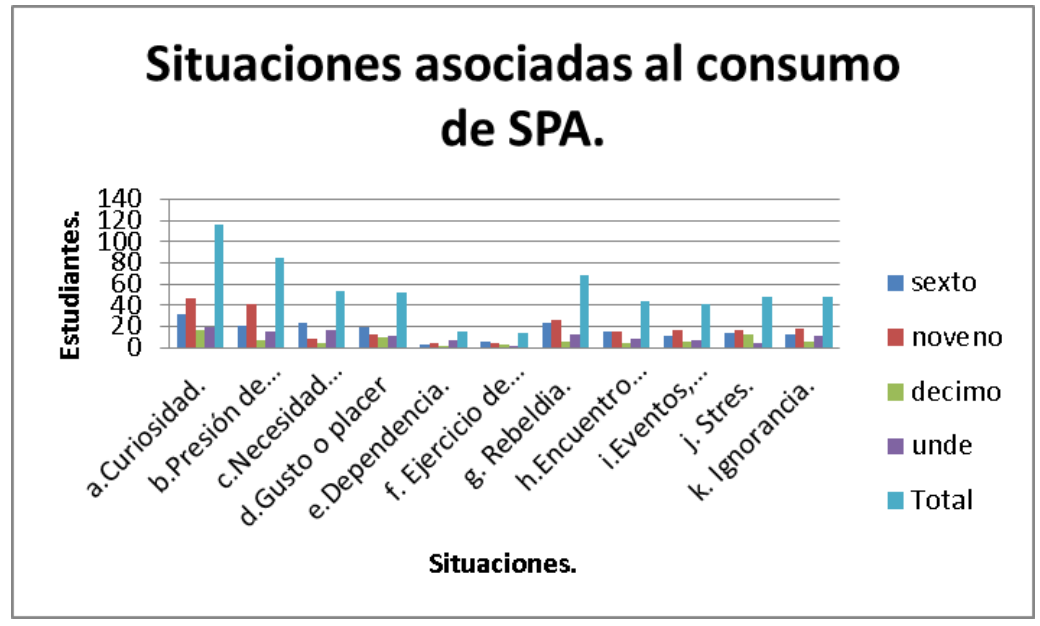

De acuerdo con estos resultados los niños y jóvenes empiezan a consumir marihuana por muchas causas, entre las más comunes curiosidad, presión de grupo, malas relaciones con sus padres y porque en los colegios no se ha asumido la responsabilidad frente al estudio y prevención del consumo de Cannabis. Las investigaciones también sugieren que los jóvenes que ya fuman cigarrillos o beben alcohol corren mayor riesgo a probarla. En otras palabras, todos los aspectos del medio en el que el niño y joven se desenvuelva son factores de riesgo para que se inicien en el mundo de las drogas.

En el anexo tres la gráfica dos presenta el género de la población encuestada de cada uno de los cursos y la gráfica tres demuestra que el $57 \%$ de los estudiantes viven en familias 
Bio - grafia. Escritos sobre la Biología y su Enseñanza. ISSN 2027

Edición Extraordinaria. p.p. 1543-1563

Memorias del VIII Encuentro Nacional de Experiencias en Enseñanza de la Biología y la Educación Ambiental. III Congreso Nacional de Investigación en Enseñanza de la Biología.

nucleares papá, mamá y hermanos, el $34 \%$ con mamá y hermanos, el $6 \%$ con papá y hermanos y el $3 \%$ con otros, esos otros son las niñas que viven entre semana en un internado cerca al colegio.

En la gráfica cuatro se puede concluir que la mayoría de estudiantes conocen gran parte de SPA. El alcohol es la sustancia que más han consumido alguna vez, seguido por el cigarrillo, la marihuana, éxtasis y pegante. Pero es preocupante el número de estudiantes que regularmente consumen alcohol, cigarrillo y marihuana; se requiere mayor atención y acompañamiento.

En la socialización de la guía (anexo 2) los estudiantes demuestran no tener claro órganos y funciones del sistema nervioso ya que lo confunden reiteradamente con el sistema circulatorio. En cuanto a las relaciones entre el sistema nervioso y los otros sistemas no las visualizan, expresan muchas confusiones, algunos creen por ejemplo, que los sentimientos y comportamientos son causados en el corazón. En la pregunta ¿Por qué el consumo de sustancias psicoactivas afecta el comportamiento de las personas? Algunas respuestas fueron: "porque las SPA tienen algo que ponen a la persona rara", "porque los ponen ansiosos, se alejan de la sociedad y los vuelve inconscientes de lo que hacen". Algunos pueden acercarse a la realidad pero en las explicaciones se quedan cortos.

En la segunda etapa se trabajaron cinco actividades en 12 encuentros. Se enfatizó sobre el trabajo en grupo, talleres, laboratorios, foros y discusiones. Es importante comentar que la búsqueda de la comprensión en la enseñanza involucró, no solamente un cambio en la concepción del proceso educativo, sino su transformación completa desde el diseño y la organización del currículo. Esta concepción diferente implica cambiar inclusive los propios conceptos desde el campo especializado del saber, hacia el contexto didáctico del campo escolar. "Es decir, transformar un conocimiento para ser enseñado y aprendido implica también conocer posibilidades y dificultades que surgen cuando se pretende que un estudiante pase de una comprensión intuitiva no escolarizada a una comprensión disciplinar" (Pogré 2012). 
Bio - grafia. Escritos sobre la Biología y su Enseñanza. ISSN 2027

Edición Extraordinaria. p.p. 1543-1563

Memorias del VIII Encuentro Nacional de Experiencias en Enseñanza de la Biología y la Educación Ambiental. III Congreso Nacional de Investigación en Enseñanza de la Biología.

En la tercera etapa es muy satisfactorio evidenciar la apropiación de conceptos y criterios relacionando el sistema nervioso con el consumo de cannabis. Gracias a la evaluación continua como proceso, por ser el motor que promueve las comprensiones, retroalimenta y anima a mejorar permanentemente no solo a nivel personal sino también en colectivo, se obtuvo niveles de comprensión de desarrollo aprendiz y maestría.

En las metas de comprensión los estudiantes lograron establecer relaciones directas entre el sistema nervioso y el funcionamiento del cuerpo y el comportamiento como también las propiedades y efectos del consumo de Cannabis, respaldados en fuentes de información, además se logró que los estudiantes cuestionaran críticamente las ideas de otros y promovieran decisiones responsables a nivel individual y colectivo. Construyeron también textos (escritos, guión de títeres, videos, plegables y cartas) para apoyar su punto de vista y sensibilizar a la comunidad del colegio para prevenir el consumo de $C$. sativa.

El reconocimiento a nivel personal, por pares, por maestros y directivos de las socializaciones y talleres que se han realizado con los diferentes estamentos de la institución ha generado un ambiente escolar en donde se evidencia la preocupación por disminuir y prevenir el consumo de cannabis.

De la experiencia se puede concluir: Para que la E.p.C garantice los resultados esperados es importante que desde el inicio de la unidad los estudiantes junto con el docente acuerden las normas y comprendan la necesidad de elaborar las sintesis conceptuales a medida que se avanza en el proceso. La evaluación debe tener criterios claros desde el comienzo de cada desempeño y siempre vinculada con la meta de comprensión.

Vincular los colegios con las universidades, no solo aporta conocimientos, sino que motiva y acompaña los procesos pedagógicos, contribuye con el mejoramiento de la educación media y afianza el optimismo para el ingreso de los educandos a la educación superior.

El estudio del sistema nervioso como pretexto para prevenir el consumo de la marihuana generó en los estudiantes interés para apropiarse de los conocimientos y tener argumentos y criterios para decir un "no" frente al consumo de cannabis y emprender el camino de la prevención con los compañeros de los diferentes cursos y jornadas. 
Bio - grafia. Escritos sobre la Biología y su Enseñanza. ISSN 2027

Edición Extraordinaria. p.p. 1543-1563

Memorias del VIII Encuentro Nacional de Experiencias en Enseñanza de la Biología y la Educación Ambiental. III Congreso Nacional de Investigación en Enseñanza de la Biología.

La propuesta didáctica que aporta a la apropiación y toma de decisiones frente a la problemática de consumo de Cannabis sativa se encuentra en la siguiente dirección. http://bioparceros.wix.com/bio-parceros\#!por-una-cultura-de-la-prevencin/ckzq

\section{BIBLIOGRAFIA}

$\checkmark$ ACEVEDO, J. (2004). Reflexiones sobre las finalidades de la enseñanza de las ciencias: educación científica para la ciudadanía. Revista Eureka sobre Enseñanza y Divulgación de las Ciencias. Vol. 1, N. Págs. 3-16.

$\checkmark$ BELEÑO, S. y DÍAZ, G. (2011) Análisis de las tendencias de drogas de abuso e impactos en la salud del individuo en países de América Latina, años 2006-2010. Universidad Nacional de Colombia. Bogotá.

$\checkmark$ ESCOBEDO, H.; JARAMILLO, R.; BERMÚDEZ, A. (2004). Enseñanza para la comprensión. Educere, 8 (octubre-diciembre). Págs. 529-534.

$\checkmark$ GIORDANO, M; POGRÉ, P. (2012) Enseñar para comprender: experiencias y propuestas para la educación superior. Buenos Aires. Ed. Teseo.

$\checkmark$ IBÁÑEZ, X.; et al. (2005). Desarrollo de actitudes y pensamiento científico a través de proyectos de investigación en la escuela. Enseñanza de las ciencias. Número extra. VII congreso.

$\checkmark$ J J

iménez, M.P (2003). La enseñanza y el aprendizaje de la biología. En Jiménez, M.P, (Coor) Caamaño A, Oñorbe A, Pedrinaci.E, De Pro. Enseñar Ciencias. (pp. 119-142) Barcelona: Grao.

$\checkmark$ MEN (1998). Lineamientos Curriculares para el área de ciencias naturales y educación ambiental. 
Bio - grafia. Escritos sobre la Biología y su Enseñanza. ISSN 2027

Edición Extraordinaria. p.p. 1543-1563

Memorias del VIII Encuentro Nacional de Experiencias en Enseñanza de la Biología y la Educación Ambiental. III Congreso Nacional de Investigación en Enseñanza de la Biología.

$\checkmark$ MEN (2004a). Estándares básicos de competencias en ciencias sociales y ciencias naturales.

$\checkmark$ MORENO, C.; et al. (2013) Efectos del consumo del THC (delta-9tetrahidrocanabinol) en el sistema nervioso, circulatorio, respiratorio, e influencia en la capacidad cognitiva, de consumidores universitarios habituales. Universidad Distrital Francisco José de Caldas.

$\checkmark$

coppetta, O. (2010). Consumo de drogas en Colombia: Características y tendencias. Bogotá D.C.: Editora Guadalupe.

$\checkmark$ SÉRÉ, M. (2002). La enseñanza en el laboratorio. ¿qué podemos aprender en términos de conocimiento práctico y de actitudes hacia la ciencia? Enseñanza de las ciencias. 20 (3). Págs.357-368.

$\checkmark$ STONE, M. la enseñanza para la comprensión, 1999, Buenos Aires.

$\checkmark$ VÁZQUEZ, A.; ACEVEDO, J.A.; MANASSERO, M.A. (2005). Más allá de una enseñanza de las ciencias para científicos: hacia una educación científica humanista. Enseñanza de las Ciencias. 4(2). 
Bio - grafia. Escritos sobre la Biología y su Enseñanza. ISSN 2027

Edición Extraordinaria. p.p. 1543-1563

Memorias del VIII Encuentro Nacional de Experiencias en Enseñanza de la Biología y la Educación Ambiental. III Congreso Nacional de Investigación en Enseñanza de la Biología.

ANEXO 1

AREA DE CIENCIAS NATURALES Y EDUCACIÓN AMBIENTAL. ENCUESTA SOBRE PREVENCIÒN Y USO DE DROGAS. CUESTIONARIO CONFIDENCIAL

Curso: Edad cumplida: Género: $F$ M Localidad

1. Personas con las cuales convive: (Marque con $X$ la respuesta)
a. Padre y hermanos
b. Madre y hermanos
c. Padre, madre, hermanos d. Otros_

\begin{tabular}{|l|l|l|l|}
\hline 2. Relaciones con la familia (marque con X) & No & $\begin{array}{l}\text { A } \\
\text { veces }\end{array}$ & $\begin{array}{l}\text { Casi } \\
\text { siempre }\end{array}$ \\
\hline $\begin{array}{l}\text { a.. Siente usted que puede contar con su familia cuando tiene } \\
\text { dificultades }\end{array}$ & & \\
\hline $\begin{array}{l}\text { b.. Se reúne con su familia para conversar sobre logros } \\
\text { personales y familiares }\end{array}$ & & & \\
\hline c.. Su familia muestra interés por sus sentimientos y afectos & & & \\
\hline d.. Siente que su familia lo quiere & & & \\
\hline e. Se reúnen en familia por lo menos una vez por semana & & & \\
\hline
\end{tabular}

3. Qué nivel de estudio le gustaría lograr y cuál cree que va lograr en su vida. 
Bio - grafia. Escritos sobre la Biología y su Enseñanza. ISSN 2027

Edición Extraordinaria. p.p. 1543-1563

Memorias del VIII Encuentro Nacional de Experiencias en Enseñanza de la Biología y la Educación Ambiental. III Congreso Nacional de Investigación en Enseñanza de la Biología.

\begin{tabular}{|l|l|l|}
\hline & Le gustaría lograr & $\begin{array}{l}\text { Cree que va lograr en su } \\
\text { vida }\end{array}$ \\
\hline a.. Secundaria & & \\
\hline b. Técnico/ tecnológico & & \\
\hline c..Universitario & & \\
\hline d..Especialización/ maestría & & \\
\hline e..Doctorado. & & \\
\hline
\end{tabular}

4. Cuáles son las DOS(2) principales acciones que realiza en su tiempo libre:
a. Practicar deporte
d. Hacer mandados
b. Leer
c. Escuchar música
e. Hacer tareas
f. Dormir
g. jugar con los amigos
h. Usar internet

5. Cuál de estos conflictos es el que le produce más angustia en su diario vivir. (Marque DOS)
a. Conflictos familiares _
b. Conflictos con los vecinos conflictos en el colegio
d. conflictos en las calles.
e. Conflictos con los amigos

c.

6. En el colegio cuál de las siguientes situaciones observa o vive con preocupación. Valore de 1 a 5 , siendo 5 la mayor dificultad y uno la menor 
Bio - grafia. Escritos sobre la Biología y su Enseñanza. ISSN 2027

Edición Extraordinaria. p.p. 1543-1563

Memorias del VIII Encuentro Nacional de Experiencias en Enseñanza de la Biología y la Educación Ambiental. III Congreso Nacional de Investigación en Enseñanza de la Biología.
a. El acoso escolar (bullyng)
b. Producción de basura

c. Robos

d. Consumo de sustancias psicoactivas

e. El mal uso de baños y bienes del colegio

7. Cuáles de las siguientes sustancias, conoce, ha consumido alguna vez, regularmente 0 nunca.

\begin{tabular}{|c|c|c|c|c|c|}
\hline sustancias & conoce & $\begin{array}{l}\text { Ha } \\
\text { consumido } \\
\text { alguna vez }\end{array}$ & $\begin{array}{l}\mathrm{Ha} \\
\text { consumido } \\
\text { regularmente }\end{array}$ & $\begin{array}{l}\text { Nunca la } \\
\text { ha } \\
\text { consumido }\end{array}$ & $\begin{array}{l}\text { Edad que } \\
\text { inicio } \\
\text { consumo }\end{array}$ \\
\hline \multicolumn{6}{|l|}{ a. alcohol } \\
\hline \multicolumn{6}{|c|}{ b. cigarrillo } \\
\hline \multicolumn{6}{|c|}{$\begin{array}{l}\text { c. ácidos } \\
\text { (LSD,Hongos) }\end{array}$} \\
\hline \multicolumn{6}{|l|}{ d. basuco } \\
\hline \multicolumn{6}{|c|}{ e. marihuana } \\
\hline \multicolumn{6}{|l|}{ f. èxtasis } \\
\hline \multicolumn{6}{|l|}{ 9. pegante } \\
\hline h. cocaina & & & & & \\
\hline
\end{tabular}

8. Con cuáles de las siguientes situaciones asocia el consumo de sustancias psicoactivas en niños y jóvenes:

a. La curiosidad

b. la presión del grupo

c. La necesidad de ser aceptado en el grupo 
Bio - grafia. Escritos sobre la Biología y su Enseñanza. ISSN 2027

Edición Extraordinaria. p.p. 1543-1563

Memorias del VIII Encuentro Nacional de Experiencias en Enseñanza de la Biología y la Educación Ambiental. III Congreso Nacional de Investigación en Enseñanza de la Biología.

d. El gusto o placer _ e. La dependencia _ f. El ejercicio de la libertad personal - g. La rebeldía

h. Encuentro con amigos_ i. Eventos especiales como las fiestas_ j. Situaciones de estrés K. Ignorancia

9. Cuántas personas que viven en su casa fuman cigarrillo. $\mathrm{N}^{\circ}$ personas

10. La información que usted tiene sobre las drogas la ha recibido principalmente de: Marque solo (DOS)
a. padres
b. amigos
c. docentes
d. internet
e. medios

de comunicación

11. A qué edad cree que se inicia a consumir sustancias psicoactivas: (Marque con X la respuesta)
a. antes de los 12 años
b. Entre los 12 y 14 años
c. Entre los 15 y 17

d. Cuando se ingresa a la universidad

e. En cualquier edad

12. Conoce estudiantes del colegio que consumen marihuana:
a. Si
b. No
si contestó Si, cuántos.
$\mathrm{N}^{\circ}$ consumidores que ud. Conoce

13. Conoce estudiantes del colegio que distribuyen -venden marihuana:
a. $\mathrm{Si}$
b. No Si contestó si, cuántos. $N^{\circ}$ distribuidoresvendedores que ud. Conoce

14. Si un compañero le ofrece marihuana para consumirla Usted: 
Bio - grafia. Escritos sobre la Biología y su Enseñanza. ISSN 2027

Edición Extraordinaria. p.p. 1543-1563

Memorias del VIII Encuentro Nacional de Experiencias en Enseñanza de la Biología y la Educación Ambiental. III Congreso Nacional de Investigación en Enseñanza de la Biología.
a. La recibe
b. La rechaza
c. La prueba
d. Le comenta a un docente
e. Le cuenta a sus padres

15. El consumo de la marihuana afecta:
a. La memoria
b. La atención
c. La coordinación y el equilibrio
d. El ritmo cardiaco

e. El aprendizaje

f. El sueño

g. El proyecto de vida

h. las relaciones familiares 
Bio - grafia. Escritos sobre la Biología y su Enseñanza. ISSN 2027

Edición Extraordinaria. p.p. 1543-1563

Memorias del VIII Encuentro Nacional de Experiencias en Enseñanza de la Biología y la Educación Ambiental. III Congreso Nacional de Investigación en Enseñanza de la Biología.

Anexo 2

COLEGIO TÉCNICO DOMINGO FAUSTINO SARMIENTO AREA DE CIENCIAS NATURALES Y EDUCACIÒN AMBIENTAL

Nombre:

Tema: Sistema Nervioso

Tópico generador: Cuál es la importancia del estudio del sistema nervioso en la prevención del consumo de $C$ sativa?

Desempeños de comprensión: Los estudiantes en forma individual explicarán algunas reacciones del cuerpo humano y la relación del sistema nervioso en estas reacciones a partir de situaciones imaginarias. También explicará por qué las sustancias psicoactivas afectan el sistema nervioso. Luego dibujarán el sistema nervioso ubicando órganos y escribiendo funciones de cada uno. Compartirán sus puntos de vista con los compañeros en cada mesa de trabajo. Posteriormente participarán de la socialización general y por último elaborarán un escrito donde expliquen tres razones por las cuales es importante el estudio del sistema nervioso en la prevención del consumo de marihuana.

Leer las siguientes preguntas y escribir su comentario

1. ¿Cuál es la relación entre el sistema nervioso y el funcionamiento del organismo? 
Bio - grafia. Escritos sobre la Biología y su Enseñanza. ISSN 2027

Edición Extraordinaria. p.p. 1543-1563

Memorias del VIII Encuentro Nacional de Experiencias en Enseñanza de la Biología y la Educación Ambiental. III Congreso Nacional de Investigación en Enseñanza de la Biología.

\begin{tabular}{|c|c|c|}
\hline $\begin{array}{l}\text { 2. Imagine las siguientes situaciones y } \\
\text { explique en cada una de ellas: }\end{array}$ & $\begin{array}{l}\text { Las diferentes } \\
\text { reacciones de su } \\
\text { cuerpo. }\end{array}$ & $\begin{array}{l}\text { De qué manera influye el } \\
\text { sistema nervioso en } \\
\text { estas reacciones? }\end{array}$ \\
\hline \multicolumn{3}{|l|}{$\begin{array}{l}\text { a. Usted va a almorzar y le sirven de } \\
\text { entrada unas empanaditas o su } \\
\text { plato preferido. }\end{array}$} \\
\hline \multicolumn{3}{|l|}{$\begin{array}{l}\text { b. En un partido de fútbol usted } \\
\text { ocupa la posición del arquero y se } \\
\text { cobra un tiro penal. }\end{array}$} \\
\hline \multicolumn{3}{|l|}{ c. En una fiesta a usted le ofrecen } \\
\hline \multicolumn{3}{|l|}{$\begin{array}{l}\text { 3. ¿Por qué el consumo de sustancias } \\
\text { psicoactivas afecta el comportamiento de } \\
\text { las personas? }\end{array}$} \\
\hline \multicolumn{3}{|c|}{$\begin{array}{l}\text { 5. Haga un dibujo del sistema nervioso ubicando sus órganos, puede completarlo con las } \\
\text { funciones. }\end{array}$} \\
\hline
\end{tabular}


Bio - grafia. Escritos sobre la Biología y su Enseñanza. ISSN 2027

Edición Extraordinaria. p.p. 1543-1563

Memorias del VIII Encuentro Nacional de Experiencias en Enseñanza de la Biología y la Educación Ambiental. III Congreso Nacional de Investigación en Enseñanza de la Biología.

Haga un escrito donde expliquen tres razones por las cuales es importante el estudio del sistema nervioso para prevenir el consumo de marihuana.

De acuerdo a su desempeño en la sesión evalúese de dos a cinco. (Escala establecida en el sistema interno de evaluación)

Anexo tres

Gráfica 2

Gráfica 3.

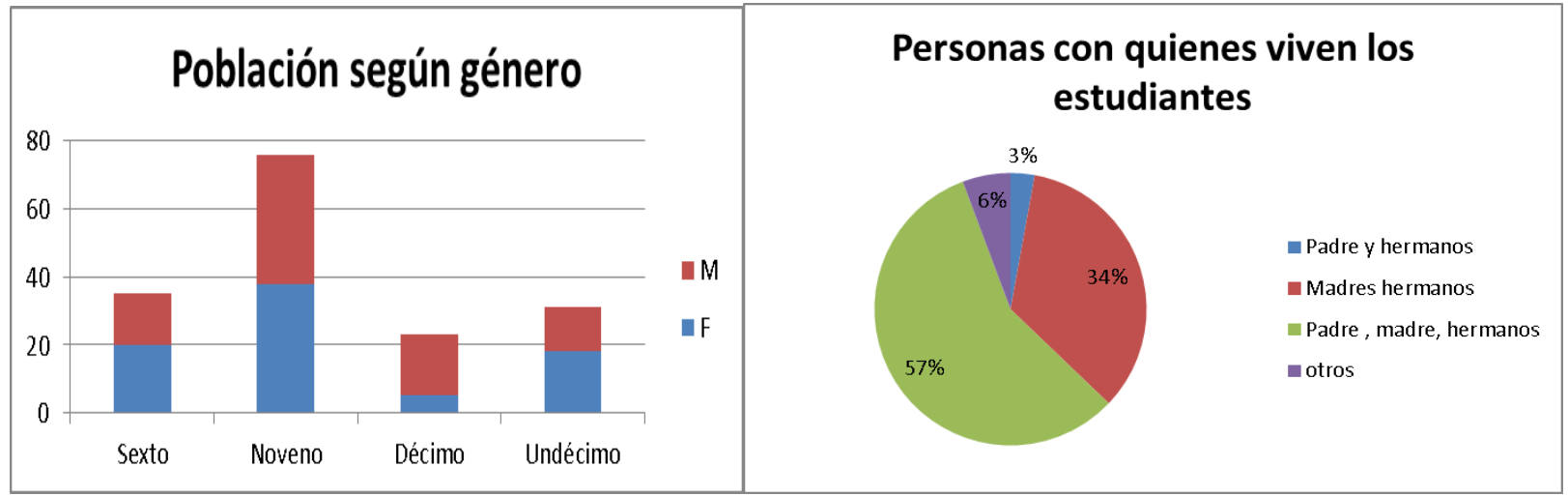


Bio - grafia. Escritos sobre la Biología y su Enseñanza. ISSN 2027

Edición Extraordinaria. p.p. 1543-1563

Memorias del VIII Encuentro Nacional de Experiencias en Enseñanza de la Biología y la Educación Ambiental. III Congreso Nacional de Investigación en Enseñanza de la Biología.

\section{Gráfica 4}

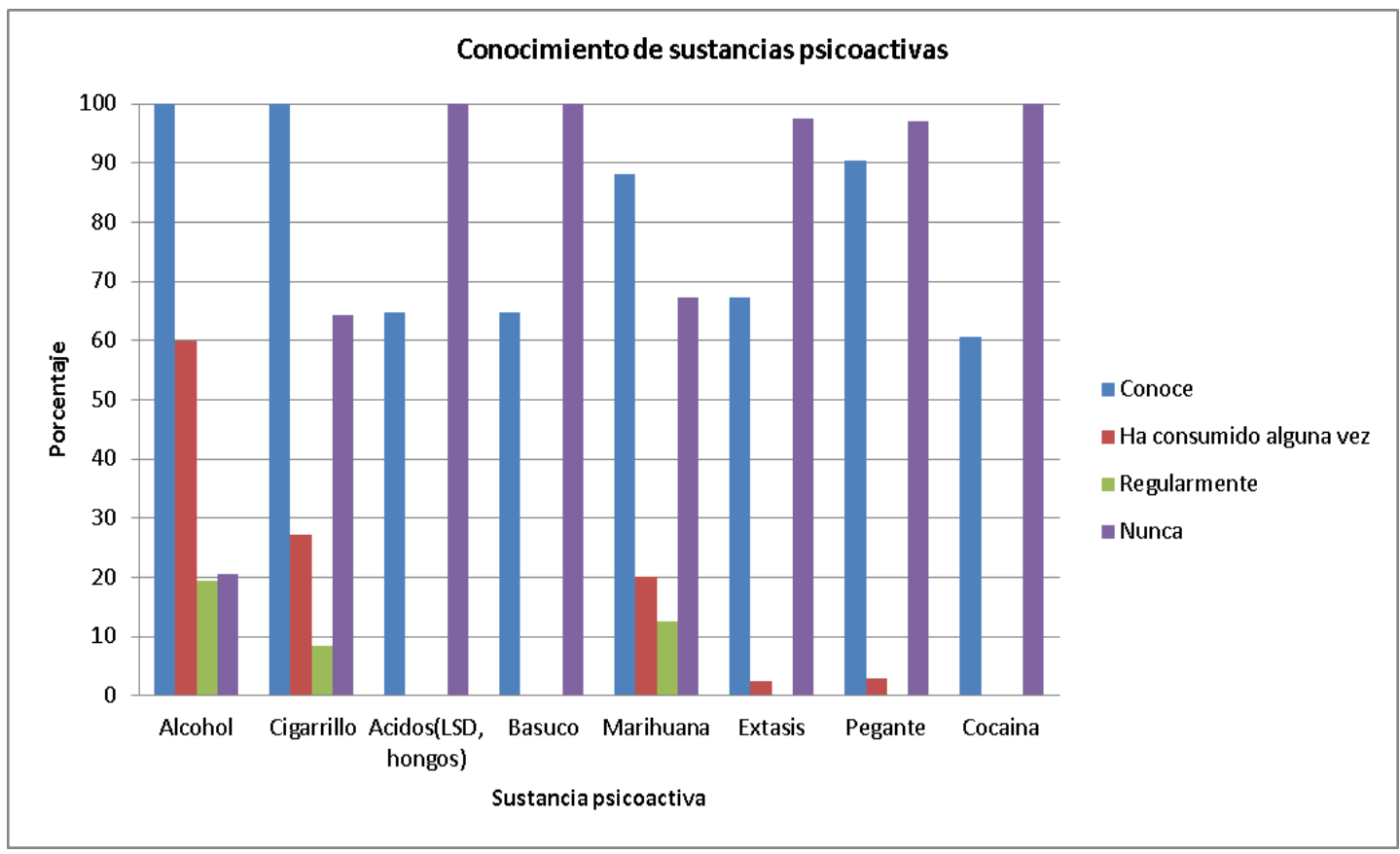

